\title{
The post-2009 influenza pandemic era: time to revisit antibody immunodominance
}

\author{
Kristien Van Reeth \\ Laboratory of Virology, Faculty of Veterinary Medicine, Ghent University, Ghent, Belgium.
}

\begin{abstract}
The current inactivated influenza vaccines rely on the induction of neutralizing antibodies against the head domain of the viral hemagglutinin (HA). The HA head contains five immunodominant antigenic sites, all of which are subject to antigenic drift, thereby limiting vaccine efficacy. Bypassing the immune system's tendency to focus on the most variable regions of the HA may be a step toward more broadly protective influenza vaccines. However, this requires a better understanding of the biological meaning of immunodominance, and of the hierarchy between different antigenic sites. In this issue of the $J C l$, Liu et al. determined the immunodominance of the five antigenic sites of the HA head in experimentally infected mice, guinea pigs, and ferrets. All three species exhibited different preferences for the five sites of the 2009 pandemic H1N1 strain. Moreover, human subjects exhibited yet a different pattern of immunodominance following immunization with the standard inactivated influenza vaccine. Together, these results have important implications for influenza vaccine design and interpretation of animal models.
\end{abstract}

Hemagglutinin: a case of immunodominance of variable over conserved epitopes

The HA protein on the surface of influenza viruses is by far the most important protein from the viewpoint of viral evolution and protective immunity. HA is composed of a globular head that contains the receptor binding site, which is essential for viral attachment to receptors on host cells, and a stalk. A second surface protein, the enzyme neuraminidase (NA), promotes release of new flu viruses from infected cells. While antibodies that bind NA and other viral proteins and $\mathrm{T}$ cell-mediated immunity contribute to protection against influenza, only antibodies that recognize the HA head are able to block viral binding and prevent entry into host cells $(1,2)$. These robust neutralizing antibodies are the main effectors of the current inactivated (or killed) influenza vaccines and they are routinely quantified by HA- inhibition (HI) assay. Unfortunately, the head region is one of the most variable regions of the $\mathrm{HA}$; therefore, vaccine strains need to be updated every few years to keep pace with the constant changes (also known as antigenic drift) in the HA of the circulating virus strains.

In 1982, it was shown that HI antibodies mainly target five physically distinct antigenic sites in the HA head of H1N1 influenza virus (3), which along with $\mathrm{H} 3 \mathrm{~N} 2$ is currently circulating in humans. Not surprisingly, these antigenic sites are hotspots for amino acid mutations that lead to antigenic drift. Each antigenic site is typically formed by amino acids from different loops of the HA and contains multiple epitopes (4). Moreover, these five antigenic sites are immunodominant compared with other antigenic regions in the HA head and stalk (ref. 4 and Figure 1). Antigenic sites in the stalk are much less variable and therefore are attractive targets for the develop-

Related Article: p. 4992

Conflict of interest: The author has declared that no conflict of interest exists.

Reference information: / Clin Invest. 2018;128(11):4751-4754. https://doi.org/10.1172/JCI124151. 
A HA antigenic structure

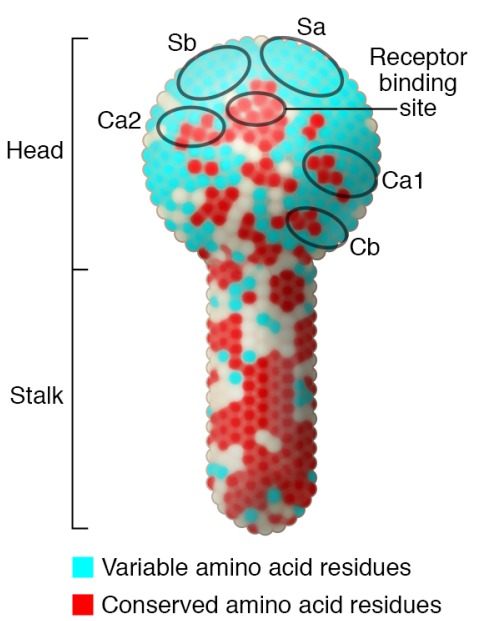

B HA antibody specificity

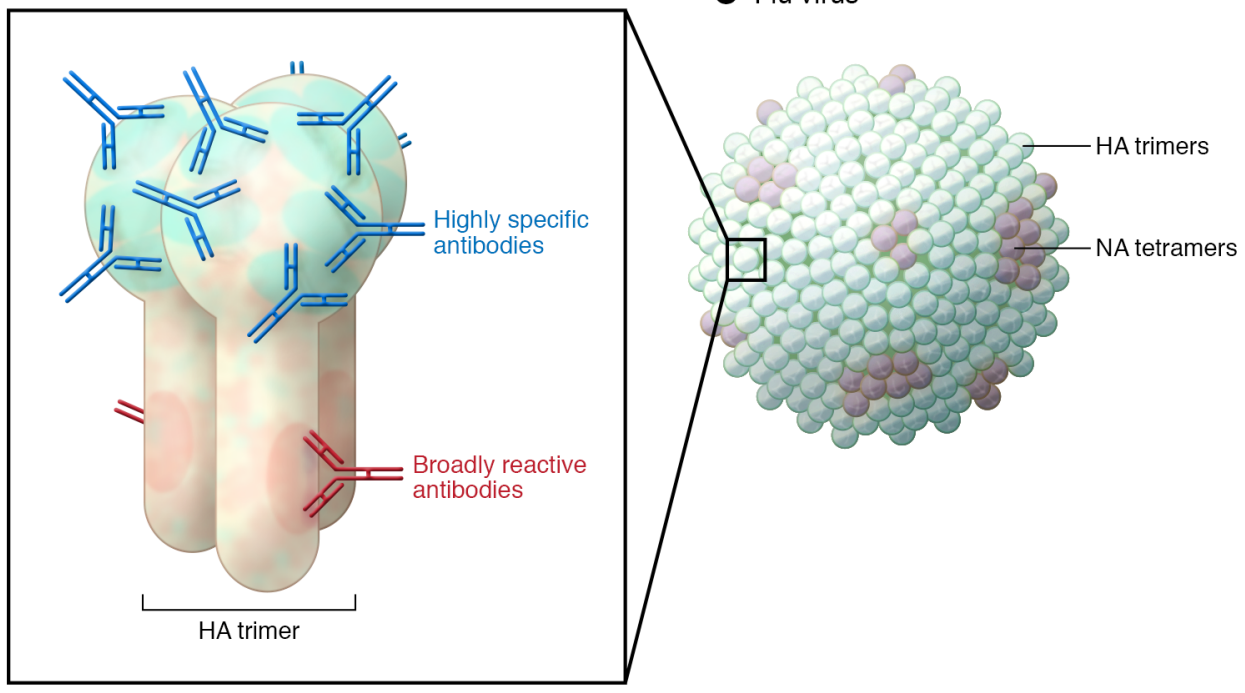

Figure 1. Schematic of influenza HA structure and variability. (A) Schematic representation of the structure of the H1N1 HA protein with its head and stalk domain. The HA head contains five immunodominant antigenic sites, designated Sa, Sb, Ca1, Ca2, and Cb. For the Sa and Sb sites, the S stands for strain-specific. These sites are most variable and are in close proximity to the receptor binding site. The $C$ sites are cross-reactive sites that are less variable between H1N1 strains and are located further downward on the HA. (B) HA head epitopes that are outside the classical antigenic sites and, in particular, epitopes in the HA stalk are more conserved among different influenza A viruses. These sites are targeted to a much lower degree by antibody-producing B cells. (C) Influenza virus particle with HA and NA protruding from the surface. The combination of HA and NA proteins determines the subtype of the virus, such as H1N1, H5N1, etc.

inance of sites among species that may account for differences in the breadth of the antibody response. The HI antibodies from ferret sera preferentially bound the highly variable Sa site, a result that may explain the narrow specificity of $\mathrm{HI}$ antibodies from this species. In contrast, HI antibodies from guinea pigs did not favor one antigenic site over another, and this lack of antigenic site preference may be responsible for the exceptionally broad anti-HA antibody response of these animals (9).

A deeper understanding of these species-specific differences in antigenic site preference will definitely help to guide the choice of animal models used for future influenza vaccine studies. Especially as the outcome of a given study will largely depend on the model chosen. Moreover, antibody immunodominance is a complex phenomenon that is as dynamic as the immune response itself. The study by Liu et al. focused only on $\mathrm{HI}$ antibodies in the circulation, which have historically been used as a correlate of protection for inactivated flu vaccines. Unlike vaccination, infection with live influenza virus also locally induces anti$\mathrm{HA}$ antibodies in the respiratory tract and these mucosal antibodies are the prime mediators of protection following infec- tion. The different type and structure and supposedly broader neutralizing capacity of mucosal antibodies compared with those in the circulation warrant further studies of immunodominance in samples of the nose and lung.

\section{The complex influenza immune repertoire in humans}

Caution should be taken when using the findings of Liu et al. to compare responses in humans and experimental animals because of differences in the route and type of immunization and the immune history, all of which can alter immunodominance hierarchies $(4,6)$. The human subjects in this study were injected intramuscularly with the standard inactivated vaccine. Moreover, these subjects have encountered numerous influenza virus infections and vaccinations with different H1N1 and other influenza viruses throughout their lifetime, and every subsequent exposure to a novel virus stimulates preexisting memory B cells to secrete antibodies to shared epitopes between old and new influenza strains. The exact exposure histories of the humans in the Liu et al. study are uncertain and differ between individuals. Differences in background immunity, as well as genetic factors (4), likely contribute to the wide variations in HI profiles observed by Liu et al. However, the HI antibodies from most of the blood donors appeared to preferentially bind the two most variable antigenic sites, Sa and Sb. Some donors that did not show an increase in total HI antibody titer after vaccination still exhibited subtle changes in antigenic site immunodominance. Liu and colleagues postulated that these changes may correlate with protection, as antibody function can vary tremendously between and even within antigenic sites (4).

One of the most important findings of Liu et al. is the detection of antibodies against nonclassical, and presumably less changeable, HA head epitopes. The authors designed a mosaic virus in which all five $\mathrm{H} 1$ antigenic sites were swapped out with the equivalent regions from an exotic $\mathrm{H} 5$ influenza virus. Antibodies against this virus were rare in prevaccination samples but were readily boosted by vaccination. This result is in accordance with several reports that infection or vaccination of adults with the 2009 pandemic H1N1 virus promotes generation of broadly neutralizing antibodies (10-12). The anti- 
bodies described in these reports were capable of neutralizing a whole series of past human $\mathrm{H} 1 \mathrm{~N} 1$ drift variants that were substantially different from the novel pandemic virus. These antibodies were mainly directed against the conserved HA stalk, but also targeted conserved epitopes in the HA head (2). Both types of antibodies are hard to induce and are not commonly found. However, they can be induced by a strain in which most of the immunodominant epitopes are different from those in previously encountered viruses, such as the 2009 pandemic virus. In this case, the rare memory B cells that react with subdominant conserved epitopes seem to be able to expand and secrete antibodies $(10,11,13)$. The HA stalk is much more conserved than the head, and it is the most promising target for a truly universal vaccine against any drift variants of human $\mathrm{H} 1 \mathrm{~N} 1$ and $\mathrm{H} 3 \mathrm{~N} 2$ viruses, as well as potentially pandemic bird flu viruses, such as H5N1, H7N9, and H9N2 (5). On the downside, anti-stalk antibodies cannot prevent viral binding to receptors and only neutralize the virus after entry into the host cell, making these antibodies less efficient. While antibodies to conserved HA head epitopes of a given H1N1 influenza strain will be ineffective against other subtypes, they would likely offer more robust protection against H1N1 drift variants due to their vastly superior potency $(2,14)$. In other words, it is likely that the breadth of protection can only be maximized at the expense of potency. The results of Liu et al., therefore, call for further investigation of the functionality of the anti-mosaic virus antibodies and their target epitopes, which also appear attractive for vaccine design. Another highly relevant question is how adjuvanted vaccines would affect antibody levels to different HA head epitopes. The addition of an adjuvant to killed influenza vaccine is a simple but effective method to boost and broaden the immune response. The adjuvanted flu vaccine, which is licensed for populations over 65 years of age, contains the oil-in-water emulsion MF59. Interestingly, MF59 has already been shown to stimulate antibodies that react with a vastly increased number of epitopes in the HA head (15).

\section{Why study immunodominance?}

The 2009 H1N1 pandemic caused a surge of vaccination studies with sequential use of antigenically different influenza viruses and/or different types of vaccine. Many of these so-called heterologous prime-boost approaches aim at tipping the balance from immunodominant to subdominant epitopes. Additionally, there is now consensus that an individual's immune history shapes the specificity of the antibody response to influenza and should be considered for future vaccine strategies (13, $16,17)$. Yet, it is poorly understood how immunodominance evolves over time and in response to exposure to different influenza virus strains. Mutant viruses, like those in the study by Liu et al., will help to address these knowledge gaps and, equally important, to compare the functionality of antibodies against dominant versus less-dominant regions of the HA. Ultimately, such studies will empower the rational design of more broadly protective influenza vaccines.

Influenza A viruses infect many other species, including wild birds, poultry, horses, and pigs, in addition to humans. The pig is a neglected but valuable animal model for human influenza (18). In addition to the anatomy and physiology of the respiratory tract, the pathogenesis and immune response to influenza are similar in pigs and in humans. Pigs are naturally susceptible to the same influenza virus subtypes as humans, and similar vaccines are used in both species. Moreover, pigs were the source of the 2009 pandemic $\mathrm{H} 1 \mathrm{~N} 1$ virus and play an important role in the influenza virus ecology. Indeed, there is bidirectional virus transmission between pigs and humans, and pigs serve as a reservoir for older and more recent human virus strains (19). Due to their large size and longer life span, pigs are well-suited for the study of mucosal immune responses and the effects of sequential immunizations (20). Hopefully, the article by Liu and colleagues will stimulate future investigations with various influenza viruses in swine and other natural hosts.

\section{Acknowledgments}

Influenza research in the author's lab is supported by the Special Research Fund of Ghent University, the Belgian Federal
Public Service for Health, Food Chain Safety and Environment, and the European Commission.

Address correspondence to: Kristien Van Reeth, Laboratory of Virology, Faculty of Veterinary Medicine, Ghent University, Salisburylaan 133, 9820 Merelbeke, Belgium. Phone: 0032.92647369; Email: kristien.vanreeth@ugent.be.

1. Dormitzer PR, et al. Influenza vaccine immunology. Immunol Rev. 2011;239(1):167-177.

2. Krause JC, Crowe JE. Committing the oldest sins in the newest kind of ways-antibodies targeting the influenza virus type A hemagglutinin globular head. Microbiol Spectr. 2014;2(5):AID-0021-2014.

3. Caton AJ, Brownlee GG, Yewdell JW, Gerhard $\mathrm{W}$. The antigenic structure of the influenza virus A/PR/8/34 hemagglutinin (H1 subtype). Cell. 1982;31(2 pt 1):417-427.

4. Angeletti D, Yewdell JW. Understanding and manipulating viral immunity: antibody immunodominance enters center stage. Trends Immunol. 2018;39(7):549-561.

5. Krammer F, Palese P, Steel J. Advances in universal influenza virus vaccine design and antibody mediated therapies based on conserved regions of the hemagglutinin. Curr Top Microbiol Immunol. 2015;386:301-321.

6. Angeletti D, et al. Defining B cell immunodominance to viruses. Nat Immunol. 2017;18(4):456-463.

7. Liu STH, et al. Antigenic sites in influenza H1 hemagglutinin display species-specific immunodominance. J Clin Invest. 2018;128(11):4992-4996.

8. Margine I, Krammer F. Animal models for influenza viruses: implications for universal vaccine development. Pathogens. 2014;3(4):845-874.

9. Nachbagauer R, et al. Defining the antibody cross-reactome directed against the influenza virus surface glycoproteins. Nat Immunol. 2017;18(4):464-473

10. Wrammert J, et al. Broadly cross-reactive antibodies dominate the human $\mathrm{B}$ cell response against 2009 pandemic H1N1 influenza virus infection. J Exp Med. 2011;208(1):181-193.

11. Li GM, et al. Pandemic H1N1 influenza vaccine induces a recall response in humans that favors broadly cross-reactive memory B cells. Proc Natl Acad Sci U S A. 2012;109(23):9047-9052.

12. Pica $\mathrm{N}$, et al. Hemagglutinin stalk antibodies elicited by the 2009 pandemic influenza virus as a mechanism for the extinction of seasonal H1N1 viruses. Proc Natl Acad Sci U S A. 2012;109(7):2573-2578.

13. Andrews SF, et al. Immune history profoundly affects broadly protective B cell responses to influenza. Sci Transl Med. 2015;7(316):316ra192.

14. Crowe JE Jr. Is it possible to develop a "univer sal" influenza virus vaccine? Potential for a universal influenza vaccine. Cold Spring Harb Perspect Biol. 2018;10(7):a029496. 
15. Khurana S, et al. MF59 adjuvant enhances diversity and affinity of antibody-mediated immune response to pandemic influenza vaccines. $S c i$ Transl Med. 2011;3(85):85ra48.

16. Li Y, et al. Immune history shapes specificity of pandemic H1N1 influenza antibody responses. J Exp Med. 2013;210(8):1493-1500.
17. Fonville JM, et al. Antibody landscapes after influenza virus infection or vaccination. Science. 2014;346(6212):996-1000.

18. Rajao DS, Vincent AL. Swine as a model for influenza A virus infection and immunity. ILAR J. 2015;56(1):44-52.

19. Nelson MI, Vincent AL. Reverse zoonosis of influenza to swine: new perspectives on the human-animal interface. Trends Microbiol. 2015;23(3):142-153.

20. Van Reeth K, et al. Heterologous prime-boost vaccination with $\mathrm{H} 3 \mathrm{~N} 2$ influenza viruses of swine favors cross-clade antibody responses and protection. NPJ Vaccines. 2017;211. 